\title{
शिमला ग्रामीण क्षेत्र की जीवन शैली में लोक संगीत का प्रभाव
}

सरिता भट्ट ${ }^{1}$

लोक संगीत का अर्थ वह संगीत है, जो लिखा नहीं गया हो और शहरी सभ्यता से अलग किसी दूर-दराज गाँव में, जहाँ आधुनिक सभ्यता अभी तक नहीं पहुँची है, जहाँ मनुष्य एक समुदाय में रहते हैं, उनकी संस्कृति या उनका संगीत 'लोक' है। 'लोक' का सामान्य अर्थ है 'जन-समुदाय'। उसमें आप, हम और गाँव व शहर में निवास करने वाले ही व्यक्ति सम्मिलित हो जाते हैं। अतः जो संगीत जन साधारण में गाया जाए वह 'लोक संगीत' है। लोक संगीत जन-साधारण की भावनाओं को व्यक्त करता है। लोक संगीत मनोरंजन का साधन माना जाता है लेकिन उसके गीतों के द्वारा सामाजिक स्थिति, रीति-रिवाज, सभ्यता-संस्कृति की झलक मिलती है।

हिमाचल प्रदेश, हिमालय पर्वत की श्रंखलाओं के बीच भारत के उत्तरी-पश्चिमी कोने में स्थित है। इसके उत्तर में जम्मू-कश्मीर, पश्चिम में पंजाब, दक्षिण में हरियाणा, पूर्व में तिब्बत है। हिमाचल प्रदेश अपने प्राकृतिक सौन्दर्य के लिए भारतवर्ष में विख्यात है। इसकी राजधनी शिमला है।

शिमला कई शहरी व ग्रामीण क्षेत्रों के मेल से बना है। शिमला ग्रामीण क्षेत्र की सभ्यता व संस्कृति बहुत विशाल है। इसमें समाज के वे सभी अनुष्ठान व कृतियां शामिल हैं, जिन्हें मानव ने अपने लम्बे इतिहास में धार्मिक, आध्यात्मिक, लौकिक, अर्थिक, राजनैतिक, सामाजिक क्षेत्र में उपयुक्त पाया है तथा आदर्श रूप में संजोए रखा है। शिमला ग्रामीण क्षेत्र के लोक जीवन, सभ्यता व संस्कृति का सुन्दर प्रतिबिम्ब हमें यहाँ के लोक संगीत में दिखाई देता है। लोक संगीत सदा संस्कृति का संगी रहा है। शिमला ग्रामीण क्षेत्र के लोक संगीत का यहां के मानव जीवन के साथ बहुत ही घनिष्ट सम्बन्ध रहा है। प्रत्येक व्यक्ति की यह स्वभाविक इच्छा रहती है कि वह अपने आन्तरिक उद्गारों को दूसरों के सामने प्रकट करे। इसलिए वह या तो वाणी का प्रयोग करता है या संकेतों को अपना साधन बनाता है।

शिमला ग्रामीण क्षेत्र के जन-साधारण लोक संगीत के द्वारा अपनी भावनाओं को अभिव्यक्त करते हैं। ग्रामीण क्षेत्र के लोक संगीत को मनोरंजन का साधन माना जाता है। लेकिन इन गीतों के द्वारा सामाजिक स्थिति, रिति-रिवाज, सभ्यता-संस्कृति की झलक मिलती है। शिमला ग्रामीण क्षेत्र के व्यक्ति लोक संगीत के द्वारा ही अपने प्राचीन को आधुनिक से जोड़े हुए हैं।

शिमला ग्रामीण क्षेत्र में लोक संगीत का प्रयोग प्रत्येक अवसर पर किया जाता है। लोक संगीत के द्वारा ग्रामीण लोग भावनाओं का सजीव चित्रण प्रस्तुत करते हैं। ग्रामीण क्षेत्र में लोक संगीत का प्रयोग मनोरंजन के अतिरिक्त देवी-देवताओं के पूजन में भी किया जाता है। ग्रामीण लोग देवता पूजन में देव लोक-गीतों को गाने के साथ-साथ देव लोक-वाद्यों का भी वादन करते हैं। ग्रामीण क्षेत्र में इन लोक वाद्यों को बजाने वाले लोगो की भी एक विशेष जाति होती है। जिन्हें ग्रामीण लोग 'तूरी' कहते हैं। केवल यही लोग देव वाद्यों का वादन करते हैं। देव वाद्यों का प्रयोग केवल देव कार्यों में ही किया जाता है। देवता पूजन या देव यात्रा में इन देव लोक वाद्यों को बजाना आवश्यक माना जाता है। ग्रामीण लोगों का यह मानना है कि यदि इन देव वाद्यों का वादन न किया जाए तो देवता प्रसन्न नहीं होते तथा पूजा अधूरी मानी जाती है।

ग्रामीण क्षेत्र में कोई भी त्यौहार, मेला, पर्व या विवाह क्यों न हो, गाँव के लोग एक जगह सम्मिलित होकर, नाचकर, गाकर और ढोलकी, ढोल आदि वाद्यों को बजाकर अपनी प्रसन्नता प्रकट करते हैं। ग्रामीण लोग समूह में ही गायन, वादन व नृत्य करते हैं तथा प्रत्येक आयु का व्यक्ति इसमें भाग लेता है। इससे ग्रामीण लोगों की एकता का भी पता चलता है। ग्रामीण लोग लोक संगीत को अपने जीवन का एक महत्वपूर्ण अंग मानते हैं तथा पीढ़ी दर पीढ़ी इसका अनुसरण करते हैं।

ग्रामीण क्षेत्रों में लोक संगीत का प्रयोग दैनिक जीवन में भी किया जाता है। यहाँ के लोग खेतों में काम करते समय लोक गीतों का गायन करते हैं। लोक संगीत को सजीव बनाए रखने में ग्रामीण महिलाओं का विशेष योगदान रहा है। वह कोई भी कार्य

1 एम.फिल. शोध-छात्रा, हिमाचल प्रदेश विश्वविद्यालय, शिमला। 
करती हों, चाहे घास काट रही हों या पशुओं को चरा रही हों, लोक गीतों को गाते ही सुनाई पड़ती हैं। कई ग्रामीण व्यक्ति पशुओं को चराते समय बाँसुरी भी बजाते हैं।

लोक संगीत न केवल सभ्यता-संस्कृति व रीति-रिवाज को दर्शात है अपितु ग्रामीण सौंदर्य को भी दर्शात है। लोक गीतों में ग्रामीण क्षेत्रों के सौंदर्य का पूर्ण रूप से वर्णन किया जाता है जिसको सुनकर मन प्रसन्न हो जाता है। अतः यह कहा जा सकता है कि लोक संगीत ग्रामीण क्षेत्रों की जीवन शेली को पूर्ण रूप से प्रभावित करता है।

लोक संगीत जनमानस का संगीत है जो इतिहास के प्रारम्भ से ही मानव को धर्मिक, सामाजिक एवं सांस्कृतिक एकता ही ओर उन्मुख करने का अत्यन्त प्रभावशाली माध्यम, सुदूर अतीत में जन्में और काल क्रम में अनेक परिवर्तनों के साथ निरन्तर प्रगति की ओर गतिशील करता रहा है।

ग्रामीण लोग, लोक संगीत का प्रयोग अपने दैनिक जीवन में भी करते हैं। गाँव के लोगों का मानना है कि लोक गीतों को गाते हुए कार्य करने से न तो समय का पता चलता है और न ही शरीर को थकान लगती है। गाँव की महिलाएँ भी कार्य करते हुए, विशेष रूप से घास काटते हुए या पशुओं को चराते समय लोक गीतों को गाती हैं। पुरूष भी लोक गीतों का पूरा आनन्द लेते हैं। वह खेतों में हल चलाते समय या कोई अन्य कार्य करते समय लोक गीतों का गायन करते हैं।

लोक संगीत ही एक मात्रा ऐसी पुंजी है जो व्यक्ति द्वारा पीढ़ी दर पीढ़ी आगे बढती जा रही है। लोक संगीत ही एक ऐसी कला है, जिसने आज भी हमारी सभ्यता व संस्कृति को जीवित रखा है। लोक संगीत मानव के जन्म से आरम्भ हो जाता है तथा उसकी मृत्यु के बाद भी वह वैसे का वैसा ही रहता है। इस प्रकार लोक संगीत मानव जीवन को पूर्ण रूप से प्रभावित करता है। मनुष्य कही भी चला जाए वह कभी भी लोक संगीत से अलग नहीं रह सकता।

निष्कर्ष यह है कि आज भी ग्रामीण क्षेत्रों में रहने वाले व्यक्ति लोक संगीत से प्रभावित होते हैं। आज भी लोक संगीत उनके सुख-दुख में उनका साथ देता है। लोक संगीत ने मनुष्य की जीवन शैली को इतना प्रभावित कर दिया है कि मनुष्य चाहे खुश हो या दु:खी, वह लोक संगीत को ही अपना माध्यम बनाता है।

\section{संदर्भ ग्रन्थ सूची}

1. आनन्द केशव, हिमाचल का लोक संगीत, नेशनल पब्लिशिंग हाऊस, दिल्ली, 1975 ।

2. चक्रवर्ती इन्द्राणी, तन तन्त्री मन किन्नरी, मध्यप्रदेश हिन्दी ग्रन्थ अकादमी, भोपाल ;म०प्र०।

3. वर्मा रामचन्द्र, मानव हिन्दी कोष पांचवा खण्ड, हिन्दी साहित्य सम्मेलन, प्रयाग 1963।

4. शरद् ओंकार, नए निबन्ध, सुलभ प्रकाशन, लखनऊ।

5. श्रीवास्तव पूर्णिमा, लोक गीतों में समाज, मंगल प्रकाशन, जयपुर, 1975।

6. हरनोट एस० आर०, हिमाचल के मन्दिर और उनसे जुड़ी लोक कथाएँ, मिनर्वा बुक हाऊस, शिमला, 1991।

7. त्रिपाठी रामनरेश, कविता कोमुदी 'ग्रामगीत', नवनीत प्रकाशन, बम्बई 1955 । 